\title{
Sodium nitrate affects myocardium in pregnant rats and their pups
}

\author{
Tatiana Sataieva $^{1}$, Igor Zadnipryany ${ }^{2}$, Walery Zukow ${ }^{3 *}$ \\ ${ }^{1}$ Department of Medical Biology, Medical Academy S.I. Georgievsky, Crimea Federal University, \\ Lenin Boulevard 5/7, Simferopol, 295006, under Russian Federation jurisdiction, \\ ${ }^{2}$ Department of Topographic Anatomy and Operative Surgery, Medical Academy S.I. Georgievsky, \\ Crimea Federal University, Lenin Boulevard 5/7, Simferopol, 295006, under Russian Federation jurisdiction, \\ ${ }^{3}$ Department of Spatial Management and Tourism, Faculty of Earth Sciences, Nicolaus Copernicus University, \\ Lwowska 1 St, 87-100 Toruń, Poland, *e-mail: w.zukow@wp.pl
}

Received: 20 March 2018 / Accepted: 12 May 2018

\begin{abstract}
The problem of pollution with nitrates and nitrites of the natural environment is very relevant nowadays. The purpose of this research was to study the morphological features of the myocardium in pregnant rats and their newborn pups exposed to chronic hemic hypoxia induced by sodium nitrite. The changes in the contractile apparatus of cardiomyocytes were atrophy and lysis of myofibrils which were most pronounced at the periphery of the cardiomyocytes, near the capillaries. A similar condition of the microcirculatory vessels accompanies absolutely all types of cardiomyopathies, regardless of the genesis of the disease. Cell-stromal relationships on the background of chronic myocardial ischemia cause destructive processes of myocardial remodeling. The final morphological outcome of prolonged hypoxic heart damage can be focal dystrophy of the myocardium, which leads to the development of chronic heart failure and the appearance of life-threatening arrhythmias.
\end{abstract}

Keywords: sodium nitrite; hypoxia; myocardium; rats; microscopy.

\section{Introduction}

The protection of human health is becoming the main prerogative of our time. As a result of anthropogenic activity mankind increasingly alienates itself from the nature and evolutionary processes that contributed to the formation of the adaptive mechanisms of the organism. A person becomes a hostage of technological progress, experiencing the pressure of other conditions created by himself (Yanvareva et al., 2006). Oxygen-containing inorganic nitrogen compounds (this includes nitric and nitrous acids, as well as their salts: nitrates, nitrites and all nitrogen oxides) are now attracting special attention. They enter the human body mainly by food and drinking water in the form of nitrates and nitrites (Gupta et al., 2000). There are other ways of getting these substances into the body (through the lungs, the skin), however, that exposure is playing a relatively minor role (Feron, 2001).

Nitrogen dioxide, entering the atmosphere with the exhaust gases of cars and then by contacting the lung epithelium forms nitric and nitrous acids, which after dissociation into nitrite and nitrate ions are able to convert hemoglobin into methemoglobin (Riobo et al., 2001). Nitrates and nitrites are also widely used as medicines - in cardiology as vasodilators acting on smooth muscle cells of veins and arteries (Feelisch \& Noack, 1987). Now it is proved that nitrites are constantly present in the body endogenously, synthesized by human itself, and the synthesis occurs at 
any level of nitrates in the diet and drinking water (Gupta et al., 2000). A number of reasons, among which the pollution of food products is primarily due to the excessive use of nitrogen fertilizers in agriculture, contributes to nitratenitrite intoxication. In this case almost all organs and tissues are affected in the human body and animals (Bacon, 1997). Nitrates and nitrites are considered capable of causing carcinogenic processes.

The problem of pollution with nitrates and nitrites of the natural environment continues to remain very relevant. Along with heavy metals and pesticides, they are the most common pollutants of the environment. Nitrate-nitrite pressure growing in many countries of the world poses a real danger to public health. A significant part of this load is associated with the supply of nitro compounds (primarily nitrates) with drinking water. According to $\mathrm{WHO}$, in some countries up to $10 \%$ of the population consumes water containing nitrates at concentrations above the permissible level (50 mg / 1 for $\mathrm{NO}_{3}$ ) (Craun et al., 1981). Nitrates that come from outside or are formed endogenously are partially converted into nitrites, which are 10 times more toxic than their predecessors. They determine the degree of danger of nitrate load on the human body. It is known that nitrates and nitrites have an adverse effect on many body systems (immune, endocrine, cardiovascular, nervous, etc.) (Yamashita et al., 1997).

Harmful effects on the body can be imposed by:

- foods containing food additives (dyes, preservatives);

- products or individual food substances (proteins, amino acids) obtained by chemical or microbiological synthesis;

- crop products obtained using irrationally applied fertilizers or irrigation water;

- livestock and poultry products obtained using improperly used feed, feed additives and preservatives (mineral and non-protein nitrogenous additives, growth stimulants, antibiotics, hormonal preparations, etc.);

- harmful substances that migrated to foods from "food equipment", utensils, inventory, packaging, packaging, etc.;

- harmful substances formed in food products (they are called impurities of endogenous origin) due to heat treatment, boiling, roasting, etc.;

- products containing metabolites of microscopic fungi (mycotoxins), formed due to non-compliance with the sanitary requirements of the technology of obtaining and storing products;

- products containing harmful substances that migrated from atmospheric air, soil, water (Yerucham et al., 1997).

The clinical picture of acute nitrite/nitrate poisoning is manifested by neurological symptoms: general weakness, severe headaches in the occipital region, lack of mobility, dizziness, darkening in the eyes, impaired coordination of motion, in severe cases - convulsive twitching, hyporeflexia (Acker \& Acker, 2010). To the mentioned above we can add that in vitro experiments, the presence of genotoxic and mutagenic properties was found in nitrites, the possibility of malignant transformation of mouse cells as a result of the action of nitrites, as well as the presence of carcinogenesis promoter properties in nitrites (Nakagawa et al., 2001). The total impact of the listed materials, in our opinion, makes it possible to seriously raise the issue of drinking water nitrites and nitrites as real factors of cancer risk (Fig. 1).

The role of nitrates $\left(\mathrm{NO}_{3}\right)$ in the formation of nitrosamines in humans was studied. In the digestive tract, under the influence of nitrate-reducing microflora, nitrates are converted into carcinogenic nitrosamines (Ger et al., 1996). The only mechanism that determines the carcinogenic risk of oral intake of nitrates and nitrites for a long time, only endogenous formation of nitroso compounds was considered (and this danger really does exist). Outside the field of view of the researchers remained other mechanisms the presence of which could be assumed, given the variety of possible biological effects caused by the action of nitrates and nitrites on the organism The studies carried out over the last two decades have confirmed this. In particular, the ability of nitrates and nitrites to modify the process of blastomogenesis was proved induced by chemical, physical and biological factors. Since in real conditions a person is exposed precisely to the combined effect of nitrates / nitrites and carcinogens, the study of the effects of such interaction seemed to be urgent (Lodovici et al., 1997). There is an opinion that NO and its derivatives, formed in tissues with inflammatory processes, can contribute to carcinogenesis. Genotoxicity of NO is associated with reactions leading to the formation of $\mathrm{O}_{2}$ and peroxides, which can directly or indirectly damage the DNA structure. A direct effect leads to the deamination of the nitrogenous bases of DNA, the formation of adducts (with the participation of peroxynitrite) and the formation of single-strand breaks in DNA. The indirect effect is explained by the interaction of reaction-active varieties of NO with other molecules, for example, with amines, thiols, and lipids. The effectiveness of the functioning of both these paths is explained by the dependence on the state of the antioxidant cell defense system or on the presence of free metal cations.

One of the manifestations of acute and chronic intoxication with nitrates and nitrites is methemoglobinemia and hemic hypoxia, which entails a variety of changes in organs and tissues. Hypoxia is one of the most important causes and consequence of a number of critical conditions. Its trigger mechanism is a violation of the supply of tissues with oxygen with the development of multi-organ failure. The degree of damage to various organs and systems may differ, which largely depends on the initial state and functional capacity of the organs and, to a lesser extent, on the 


\section{TOXIC MECHANISMS OF NITRATES}

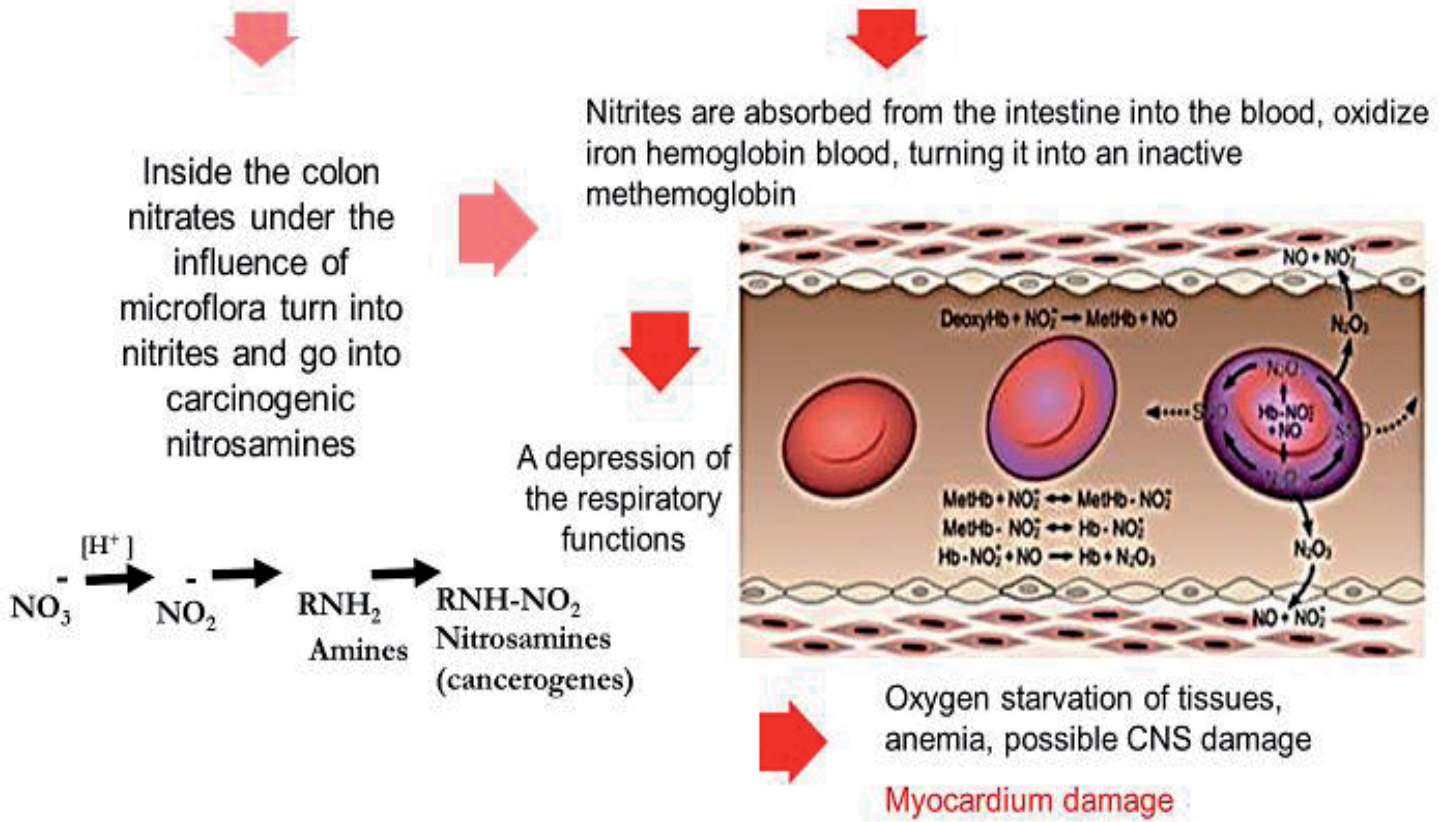

Figure 1. Toxic mechanisms of nitrates

primary damaging factor (Kloner et al., 2001). It is obvious that the results of resuscitation and intensive therapy of any critical condition are largely determined by the timeliness and adequacy of antihypoxic correction. The diversity and severity of the arising disorders in the human body dictate the need for the development of comprehensive measures and the improvement of therapeutic preventive measures aimed at reducing the consequences of nitrite intoxication.

There is no doubt that the origins of many chronic, disabling or fatal pathological conditions in adults, including diseases of the cardiovascular system, begin in the periand neonatal period, and some diseases of the neonatal, thoracic and older age represent a prolonged pathology embryo and fetus (Knobeloch et al., 2000). In this connection, questions of the state of the cardiovascular system in newborns after chronic intrauterine, intranatal or combined (perinatal) hypoxia (transient posthypoxic myocardial ischemia) are still relevant (Simanonok, 1996). It is known that hypoxia of the fetus leads to a violation of autonomic regulation of coronary vessels, a deterioration in energy metabolism with a sharp decrease in the formation of macroergic compounds in mitochondria of cardiomyocytes and sinus node cells (Patterson \& Zhang, 2010). However, such studies do not disclose the causal relationship between the influence of hemic hypoxia of matter and the formation of myocardial dysfunction in a newborn (Kloner \& Jennings, 2001).
The purpose of this research was to study the morphological features of the myocardium in pregnant rats and their newborn pups exposed to chronic hemic hypoxia.

\section{Materials and methods}

The experimental part was performed on 12 females of three-month-old white Wistar rats weighing 180200 grams. All manipulations on animals were carried out in strict accordance with the "Rules for Conducting Qualitative Clinical Trials in the Russian Federation" (approved by the Ministry of Health of the Russian Federation and enacted on January 1, 1999), the provisions of the Helsinki Declaration (2000) and the recommendations contained in the European Community Directives (No 86/609EC). Council Directive 86/609/EEC of 24 November 1986 on the approximation of laws, regulations and administrative provisions of the Member States regarding the protection of animals used for experimental and other scientific purposes (Council Directive 86/609/EEC, 1986). For the induction of pregnancy, each female was supplied with mature mongrel rats from males at a rate of two males per four females. Throughout the pregnancy and for 21 days (the end of the period of breastfeeding) after the delivery, females were injected intraperitoneally with sodium nitrite $\mathrm{NaNO}_{2}$ daily, at a dose of $5 \mathrm{mg} / 100 \mathrm{~g}$ of weight (dose 
causing moderate hypoxia). During the first day of birth we slaughtered adult rats and pups fed by the above-mentioned females and after carrying out thoraco- and pericardiotomy under ether anesthesia, the heart was removed and immediately placed in a cardioplegic solution $(0.9 \% \mathrm{KCl}$ at a temperature of $0^{\circ} \mathrm{C}$ ) than the cardiac arrest in diastole was achieved. For histological examination, samples of the left ventricular tissues of the hearts of females and pups were fixed in $10 \%$ neutral formalin and, after appropriate wiring, were filled into paraffin. Histological sections were made with a thickness of 10-15 $\mu \mathrm{m}$, stained with hematoxylineosin and examined with the Olympus CX-31 microscope (Japan). Preparation of material for the ultramicroscopic examination was carried out according to the standard procedure (Zadnipryany et al., 2017).

\section{Results and discussion}

It was revealed that similar pathomorphological changes were observed in the myocardium of the experimental females and newborns. Regardless of the diameter of the blood vessels in the myocardium of females who suffered pregnancy on the background of the influence of chronic hemic hypoxia the phenomena of hemodynamic disturbances were widely observed manifested by perivascular edema, venous plethora, desolation and spasm of arterioles. In this case the nuclei of endotheliocytes in spasmodic arterioles due to edema of the cytoplasm were visually displaced closer to the lumen of the vessels. In the perivascular space, thin layers of connective tissue were defined, as well as moderate circular cell proliferation (Fig. 2).
The microcirculatory unit of the vascular component of the myocardium was full-blooded, often the phenomena of stasis of erythrocytes was observed in capillaries, precapillaries and arterioles. Precapillary edema was recorded in almost all females, and the degree of its expression varied significantly. In some capillaries endotheliocyte rounding was observed, manifested by the bulging of the endothelial cell nuclei into the capillary lumen, which was regarded as a compensatory reaction, which, however, reduced their metabolic functions and the nourishment level of the local cardiomyocytes, which was confirmed by the slide-staining data.

Cardiomyocytes of females demonstrate a pronounced polymorphism of the nuclei. Most frequently the shape of the nuclei of cardiomyocytes was oval, with indistinct wave-like contours, which may indicate a violation of the heart rhythm. The chromatin of such nuclei was condensed and located mainly along the periphery of the nucleus. The sarcoplasm of such cardiomyocytes contained a fuchsinophilic substrate, an indicator of ischemic damage that accumulated near the nuclei, and also in the form of uneven thin interlayer interstitial layers.

Changes in the microcirculatory vessels and interstitium in newborn pups as well as in females differed in polymorphism. Arterioles and small intramural arteries were quite rare and looked significantly altered: their walls were thickened, homogenized. The lumen in such vessels was sliced or not, apparently, due to spasm and edema of endotheliocytes (Fig. 3). The full-blooded capillaries had unevenly colored contours, the distance between the capillaries and cardiomyocytes was significantly enlarged, which naturally made it difficult to transport substrates

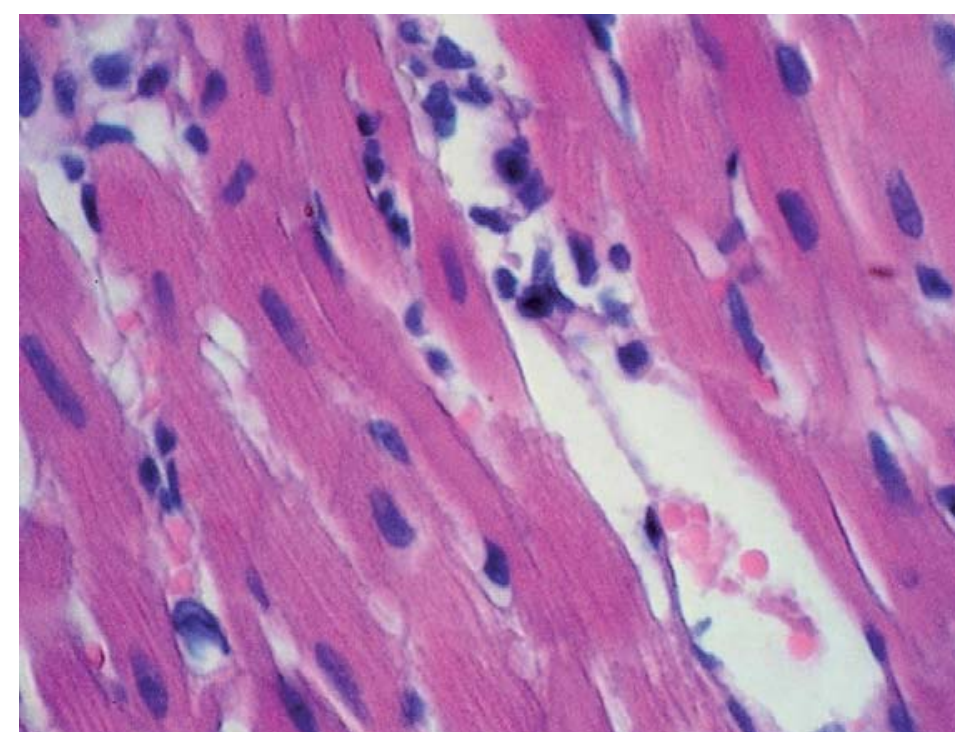

Figure 2. Vivid perivascular edema, desiccation and spasm of arterioles, mildly expressed perivascular growth of unformed loose connective tissue. Hematoxylin-eosin staining. Magnification x 400 
and oxygen from the bloodstream directly to the working cells. In addition, almost all the vessels were enveloped in thin fibers of immature loose connective tissue, which indicated the initial stages of myocardial remodeling. In some sections, diapedesis of erythrocytes was revealed in the interstitium, which indirectly indicated disturbance of tissue respiration processes. Furthermore the cardiomyocytes of the newborns looked either unevenly hypertrophic or sharply thinned. Near some nuclei or inside them there were oxyphilic inclusions, similar to apoptotic bodies. Most of the cadiomyocytes were separated from each other due to interstitial edema, and also had contracture changes (Fig. 3).

According to transmissive electron microscopy data (TEM), endotheliocytes of capillaries of pregnant rats after hypoxia were thinned, the level of micropinocytosis in them was reduced. Some capillaries were marked by tortuosity of the luminal surface (Fig. 4).

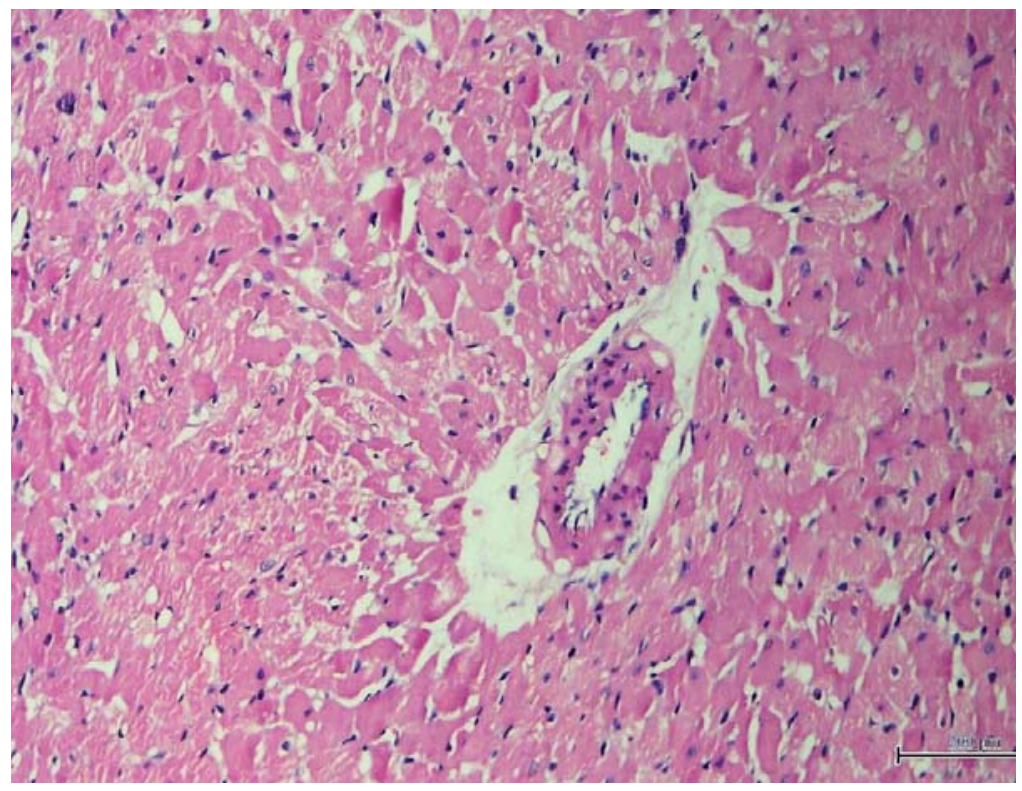

Figure 3. Perivascular and interstitial edema, collagenogenesis in perivascular space. Contractual changes and focal fragmentation of myocardial muscle fibers Hematoxylin-eosin staining. Mag. x 80

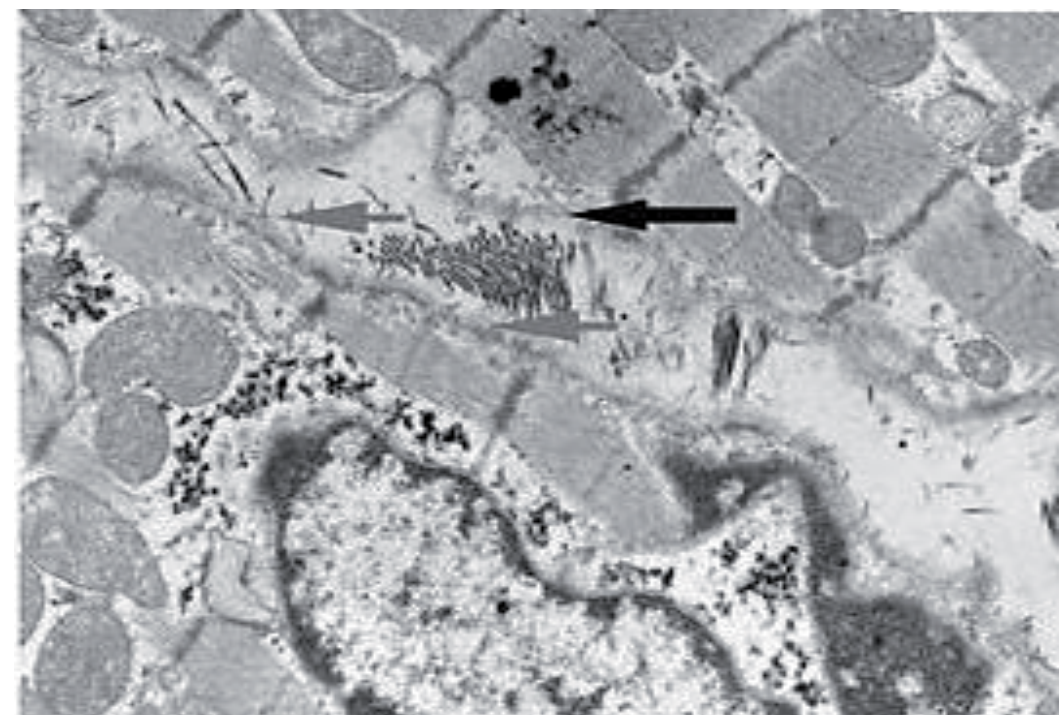

Figure 4. The walls of the full-blooded capillary are osmiophilic. Pericapillary space is widened and filled with a fibrous component of loose connective tissue (arrows). Small-focal and diffuse lysis of myofibrillar bundles. TEM. Mag. x 12,000 
The lumen of the majority of the vessels was compensatively expanded, slugging was observed, as well as the adhesion of the formed blood elements to the wall of the capillaries, which appeared, apparently, due to disruption of the basement membrane structure. The space between the capillary membrane of the capillaries and the cardiomyocytes (adventitial layer) was mainly enlarged and filled predominantly with a fibrous component of loose connective tissue (in which collagen fibers prevailed), less often electron-transparent amorphous matter of weak electron density (in which individual fibrous structures were located such as collagen fibers). In the latter case, the pericapillary space was highly enlightened, it revealed areas of a substance whose weak electron-optical density is identical to the plasma density in the capillary lumen. The outlines of these sections were not clear. The walls of most capillaries were sharply osmiophilic, apparently impregnated with plasma. Cardiomyocytes, which were located near similar vessels, were characterized by fine-focal and diffuse lysis of myofibrillar bundles, the so-called "melting" of myofibrils. To a greater extent, I-bands were lysed, in which thin (actin) filaments were located. Myofibrils became less dense, many sarcomeres showed voids, in some cases total lysis of myofilaments within the sarcomere was noted. Significant destruction was also caused by sarcomeres in the area of intercalary discs and in the perinuclear zone.

The examonation of myocardial electronograms of newborn rat pups revealed that the cytoplasm of the en- dothelial cell cytoplasm in one and the same capillary is sharply enlightened in some cells, which indicates the swelling of the cytoplasm, while in other cells it became electronically optically dense (Fig. 5).

If the matrix of the endothelial cell was enlightened, then in the cytoplasm, single micropinocytosis vesicles of a closed type of small size, mainly located along the free and basal margins of the cytoplasm were visualized. When the matrix of the cytoplasm of the endothelial cell is compacted, the micropinocytosis vesicles of various types are located along the basal and free margins of the cytoplasm as well as in the cytoplasm itself. Moreover, larger pinocytosis vesicles were often present in the cytoplasm of dark endotheliocytes. Small vesicles had electronically optically weakly dense contents. Large vesicles, on the other hand, were electronically optically transparent. The basal membrane of the capillaries looked enlarged, multilayered. Diapedesis hemorrhages were often detected. as well as nonessential for the myocardium fenestrated capillaries, which appeared, apparently due to partial destruction of endotheliocytes. In the cytoplasm of endothelial cells numerous folds and bays were observed on the luminal surface of the cells, sometimes with the detachment of protruding parts of the endotheliocytes into the lumen of the vessel. In the endotheliocytes and neighboring cardiomyocytes, a large number of mitochondria with an enlightened matrix and destroyed cristae also occurred, which indicated the development of mitochondrial dysfunction.

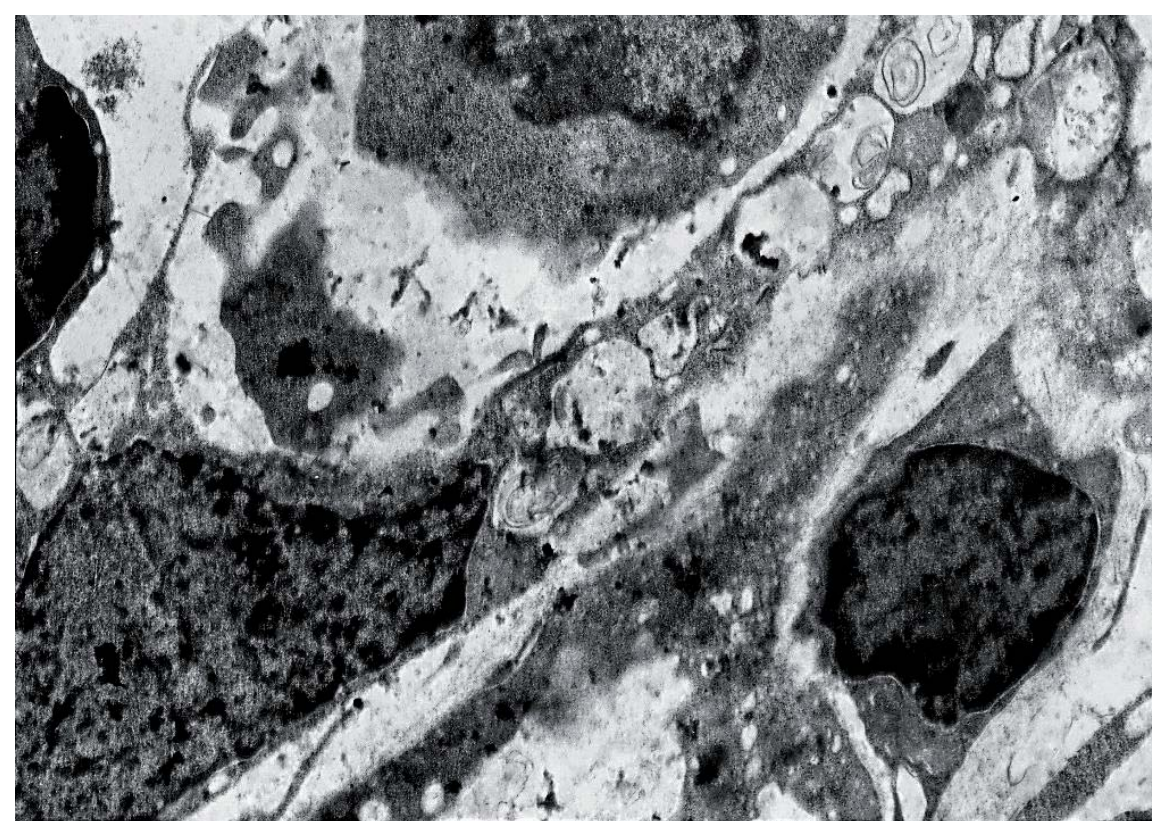

Figure 5. The electron-dense endotheliocyte with micropinocytosis vesicles, which contain layered contents, the nucleus of the endotheliocyte has pointed invaginate. Destruction of the basal membrane. In the lumen of the capillary there is a large number of leukocytes. TEM. Mag. x18000 
According to the authors' observations, the changes in the contractile apparatus of cardiomyocytes - atrophy and lysis of myofibrils - were most pronounced at the periphery of the cardiomyocytes, near the capillaries. A similar condition of the microcirculatory vessels accompanies absolutely all types of cardiomyopathies, regardless of the genesis of the disease. Cell-stromal relationships on the background of chronic myocardial ischemia cause destructive processes of myocardial remodeling. The final morphological outcome of prolonged hypoxic heart damage can be focal dystrophy of the myocardium, which leads to the development of chronic heart failure and the appearance of life-threatening arrhythmias.

Thus, the analysis of the morphology of the microvasculature of the vascular bed in pregnant and neonatal rats who underwent hemic hypoxia revealed similar dystrophic and destructive changes, which are more severe in neonatal rats. It is well known that as a highly specialized tissue, the myocardium receives practically all substrates from the blood; the synthetic ability of these cells seems to be minimal. Violation of the delivery of substrates and oxygen in conditions of hemic hypoxia, which is exacerbated by dysfunction of the circulatory pathway, inevitably affects the morphological state of cardiomyocytes, their contractility.

Early diagnosis and timely correction of the revealed pathological changes from the heart in children of the first year of life can become the most important factor in reducing the frequency and severity of cardiovascular pathology in newborns and pregnant women. A new approach in the therapy of hypoxic damage is the development of a class of pharmacological preparations of antihypoxants capable of reducing or eliminating manifestations of hypoxia, by maintaining the minimum permissible energy metabolism in the cell, especially in the early post-hypoxic period.

\section{Conclusions}

1. Chronic hemic hypoxia has a vivid pathological effect on the myocardium of pregnant and newborn rats, the effect of hypoxia on the myocardium of pups was more pronounced.

2. The main morphological signs of nitrite damage to the myocardium in pregnant rats and their pups were present in the form of mixed dystrophy, edema and lesions of endotheliocytes and contractile cardiomyocytes, lysis of myofibrils, and the appearance of over-contractions. Hemodynamic disturbances were observed everywhere, those were perivascular edema, capillary plethora, desolation and spasm of arterioles. In the perivascular space thin interlayers of connective tissue were defined and that further exacerbated the transport of substrates and oxygen from the bloodstream to the working cells under hypoxic conditions.

3. Structural changes in the microcapillaries due to the additional ischemia aggravate pathological changes in the contractile apparatus of cardiomyocytes under hypoxia, since atrophy and lysis of myofibrils were most pronounced near damaged capillaries.

4. The described changes can promote the development of myocardial insufficiency.

\section{References}

Acker T. \& Acker H., 2010, Cellular oxygen sensing need in CNS function: physiological and pathological implications. J. Exp. Biol. 2007: 3171-3188.

Bacon R., 1997, Nitrate preserved sausage meat causes an unusual food poisoning incident. Commun. Dis. Rep. CDR Rev. 7(7): 45-47.

Craun G.F., Greathouse D.G. \& Gunderson D.H., 1981, Methaemoglobin levels in young children consuming high nitrate well water in the United States. Int. J. Epidemiol. 10: 309-317.

Council Directive 86/609/EEC, 1986, On the approximation of laws, regulations and administrative provisions of the Member States regarding the protection of animals used for experimental and other scientific purposes. (http://eur-lex.europa.eu/legal-content/ EN/TXT/?uri=CELEX:31986L0609) [Accessed 19.12.2016].

Feelisch M. \& Noack E., 1987, Nitric oxide (NO) formation from nitrovasodilators occurs independently of hemoglobin or non-heme iron. Eur. J. Pharmacol. 142: 465-469.

Ger J., Kao H., Shih T.S. \& Deng J.F., 1996, Fatal toxic methemoglobinemia due to occupational exposure to methyl nitrite. Clin. Med. J. 57: 578.

Gupta S.K., Gupta R.C., Seth AK., Gupta A.B., Bassin, J.K. \& Gupta A., 2000, Methemoglobinaemia in areas with high nitrate concentration in drinking water. Nat. Med. J. India 13(2): 58-61.

Feron V.J., Arts J.H., Kuper C.F., Slootweg P.J. \& Woutersen R.A., 2001, Health risks associated with inhaled nasal toxicants. Crit. Rev. Toxicol. 31: 313-347.

Kloner R.A., Arimie R.B., Kay G.L., Cannom D., Matthews R., Bhandari A., Shook T., Pollick C. \& Burstein S., 2001, Evidence for stunned myocardium in humans: a 2001 update. Coron. Artery Dis. 12(5): 349-356.

Kloner R.A. \& Jennings R.B., 2001, Consequences of brief ischemia: stunning, preconditioning, and their clinical implications: part 1. Circulation 104(24): 2981-2989.

Knobeloch L., Salna B., Hogan A., Postle J. \& Anderson H., 2000, Blue babies and nitrate-contaminated well water. Environ Health Perspect 108: 675-678. 
Lodovici M., Casalini C., Briani C. \& Dolara P., 1997, Oxidative liver DNA damage in rats treated with pesticide mixtures. Toxicology 117: 55-60.

Nakagawa D., Ohshima Y., Takusagawa M., Ikota N., Takahashi Y., Shimizu S. \& Ozawa T., 2001, Functional Modification of Cytochrome $\mathrm{C}$ by Peroxynitrite in an Electron Transfer Reaction. Chem. Pharm. Bull. 12(12): 1547-1554.

Patterson A.J. \& Zhang L., 2010, Hypoxia and fetal heart development. Curr. Mol. Med. 10(7): 653-666.

Riobo N.A., Clementi E., Melani M., Boveris A., Cadenas E., Moncada S. \& Poderoso J.J., 2001, Nitric Oxide Inhibits Mitochondrial NADH: Ubiquinon Reductase Activity through Peroxynitrite Formation. Biochem. J. 359: 139-145.

Simanonok J.P., 1996, Non-ishemic hypoxia of the arterial wall is primary cause of atherosclerosis. Med. Hypoteses 46 (2): 155-161.
Yamashita T., Ando Y., Sacashita N., Hirayama K., Tanaka Y., Tashima K., Uchino M. \& Ando M., 1997, Role of nitric oxide in the cerebellar degeneration during methylmercury intoxication. Biochim. Biophys. Acta 1334(2-3): 303-311.

Yanvareva I.N., Baranova T.I., Kovalenko R.I. \& Mitrofanova A.V., 2006, Evolutionary determined adaptation to hypoxia and human health. VIII World Congress Int. Society for Adaptive Medicine, Moscow, Russia: 1718.

Yerucham I., Shiosberg A., Hanji V., Bellaiche M., Marcus M. \& Liberboim M., 1997, Nitrate toxicosis in beef and dairy cattle herds due to contamination of drinking water and whey. Vet. Hum. Toxicol. 39 (5): 296-298.

Zadnipryany I.V., Tretiakova O.S., Sataieva T.P. \& Zukow W., 2017, Experimental review of cobalt induced cardiomyopathy. Russian Open Medical Journal 6(1): 1-4. 